Olga Blazekova - Maria Vojtekova*

\title{
USING OF PARALLEL COORDINATES IN FINDING MINIMUM DISTANCE IN TIME-SPACE
}

\begin{abstract}
Airspace domain may be represented by a time-space consisting of a three-dimensional Cartesian coordinate system and time as the fourth dimension. A coordinate system provides a scheme for locating points given its coordinates and vice versa. The choice of coordinate system is important, as it transforms data to geometric representation. Visualization of the three and more dimensional data on the two-dimensional drawing - computer monitor is usually done by projection, which often can restrict the amount of information presented at a time. Using the parallel coordinate system is one of possibilities to present multidimensional data. The aim of this article is to describe basics of parallel coordinate system and to investigate lines and their characteristics in time-space.
\end{abstract}

Keywords: parallel coordinates, time-space, air traffic control

\section{Introduction}

During the peak air travel times in the United States, there are about 5,000 airplanes in the sky every hour. This translates to approximately 50,000 aircraft operating every day. How are these aircraft kept from colliding with each other? How does air traffic move into and out of an airport? A collision between aircrafts is one of the most sudden and catastrophic transportation accidents. Ensuring the highest level of passenger safety is a key goal for air traffic control (ATC) authorities. Air traffic controllers must coordinate all movements of thousands of aircrafts, keep them at safe distances from each other, direct them during the takeoff and landing aircrafts from and to airports, direct them around bad weather and ensure that traffic flows smoothly with minimal delays [1]. Many new conflict detection, resolution and visualization tools have been developed to support the needs of controllers. Data visualization tools portray the volume data in visual schemes that facilitate the human operator discovering the relationship that would not be apparent in tables and graphs. Visualization of the three and more dimensional data on the two-dimensional drawing - computer monitor is only possible by projection, which often can restrict the amount of information presented at a time. Using the parallel coordinates represents one of options to visualize the multidimensional data.

Airspace domain constitutes time-space consisting of threedimensional Cartesian coordinate system and time as the fourth dimension. The $x$-axis represents altitude where the positive $x$ direction is of the higher elevation and the negative $x$ direction is of the lower elevation. The $y$-axis represents the east and west directions, the positive $y$ direction points east and the negative $y$ direction points west. The $z$-axis represents north and south with the positive $z$ direction pointing north and the negative $z$ direction pointing south. In the parallel 4 coordinate plot, each dimension (variable) corresponds to an axis, and the 4 axes are organized as uniformly spaced vertical lines. A data element in the 4-dimensional space manifests itself as a connected set of points, one on each axis. Points lying on a common line or plane create readily perceived structures in the image.

Parallel coordinates is a widely used visualization technique for multidimensional objects and multivariate relations. The system was invented by Philbert Maurice d'Ocagne in 1885 [2] and were independently re-discovered and popularized by Al Inselberg in 1959 and systematically has been developed as a coordinate system starting from 1977. The parallel coordinates methodology was used when the new air traffic control system in United States was formed around 1985 - US patent \# 4,823,272, p. 100 [3]. Using of parallel coordinates for visualization of the high-dimensional data set is shown in [4-6]. The authors of [7] were concentrated on ordering and positioning of the coordinate axes. Authors of [8] compared scatterplots and parallel coordinate plots as visualization methods. A method for mobility analysis based on the parallel coordinates is presented by [9]. Application of the parallel coordinates in geographic visualization is shown in [10]. Moreover, the parallel coordinates coupled with statistical analysis can be used for more effective knowledge discovery and confirmation in complex, environmental data sets [11].

\section{Parallel coordinates in space-time}

Many applications are using the space-time which regards space as consisting of three dimensions, and time asconsituting the fourth dimension. The parallel coordinates are based on projective geometry and duality of points and hyperplanes in the $n$-dimensional space. Hyperplane in the projective space $\mathbb{P}^{4}$ is the three-dimensional space and a line is the intersection of three nonparallel hyperplanes [1]. Let a space-time have coordinates $t, x_{1}, x_{2}, x_{3} ; t$ is the time axis, $x_{1}, x_{2}, x_{3}$ axes assign three dimensional position of a point.

\footnotetext{
* Olga Blazekova, Maria Vojtekova

Faculty of Operation and Economics of Transport and Communications, University of Zilina, Slovakia

E-mail: olga.blazekova@fpedas.uniza.sk, maria.vojtekova@fpedas.uniza.sk
} 


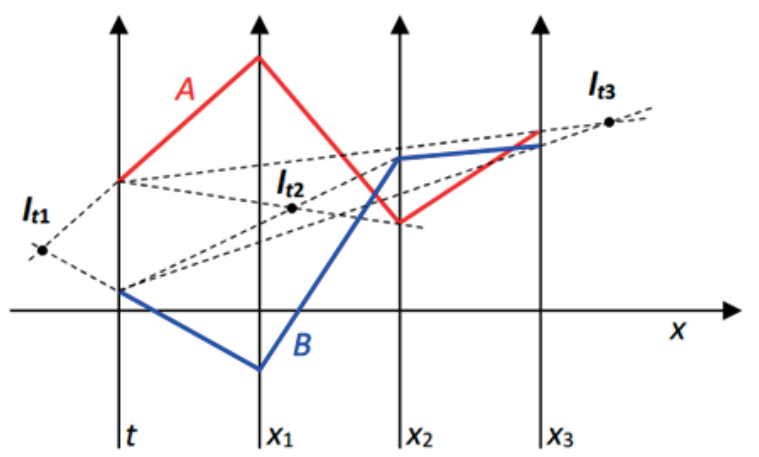

Figure 1 Representation of the line $l=A B$ in parallel coordinates $t, x_{1}, x_{2}, x_{3}$

\subsection{Representation of a line in space-time}

One can say that the line $l$ is the set of points (specified by 4 -tuples $\left.\left(t, x_{1}, x_{2}, x_{3}\right)\right)$ satisfying a set of the three linearly independent linear equations (at least one of coefficients $a_{t}, a_{1}, a_{2}, a_{3}$ must be nonzero and so on):

$$
\begin{aligned}
& a_{t} t+a_{1} x_{1}+a_{2} x_{2}+a_{3} x_{3}+a_{0}=0 \\
& l: b_{t} t+b_{1} x_{1}+b_{2} x_{2}+b_{3} x_{3}+b_{0}=0 \\
& c_{t} t+c_{1} x_{1}+c_{2} x_{2}+c_{3} x_{3}+c_{0}=0
\end{aligned}
$$

and one wants to find a suitable representation of the line $l$.

After some arrangements and using equivalent matrix operations, one obtains (with the exception of some special cases but here is being dealt with the general situation):

$$
\left(\begin{array}{lllll}
a_{1} & a_{2} & a_{3} & a_{t} & a_{0} \\
b_{1} & b_{2} & b_{3} & b_{t} & b_{0} \\
c_{1} & c_{2} & c_{3} & c_{t} & c_{0}
\end{array}\right) \sim\left(\begin{array}{rrrrr}
-1 & 0 & 0 & m_{1} & q_{1} \\
0 & -1 & 0 & m_{2} & q_{2} \\
0 & 0 & -1 & m_{3} & q_{3}
\end{array}\right) .
$$

So, one can write the convenient form of the line representation:

$$
\begin{aligned}
l_{t, 1}: x_{1} & =m_{1} t+q_{1} \\
l: l_{t, 2}: x_{2} & =m_{1} t+q_{2} . \\
l_{t, 3}: x_{3} & =m_{3} t+q_{3}
\end{aligned}
$$

Each hyperplane $l_{t, 1}, l_{t, 2}, l_{t, 3}$ is represented in the parallel coordinates by a point, so the line $l$ is represented by three points, Figure 1.

Generally, each equation is containing a pair of adjacently indexed variables. In the $x_{i-1}, x_{i}$ plane, the relation labeled $l_{i-1, i}$ is a line, and by point $\leftrightarrow$ line duality it can be represented by the point $l_{i-1, i}$. Many applications are using the $t$ variable as a basic one, so it is more comfortable to use tuples $\left(t, x_{1}\right),\left(t, x_{2}\right),\left(t, x_{3}\right)$.

Geometrically: aline $l$ is defined by two points $A\left(t^{A}, x_{1}^{A}, x_{2}^{A}, x_{3}^{A}\right)$, $B\left(t^{B}, x_{1}^{B}, x_{2}^{B}, x_{3}^{B}\right)$ and one can obtain points $l_{t, i}$ as intersections of relevant line (line segments): $l_{t, i}=t^{A} x_{i}^{B} \cap t^{B} x_{i}^{A}, i=1,2,3$ (Figure 1).

If the distance between each pair of adjacent parallel axes $t, x_{1}, x_{2}, x_{3}$ is taken as one unit and Cartesian plane coordinates $x, y$ are defined (axis $x$ is horizontal line and $y=t$ axis), then the homogeneous coordinates of points $l_{t, i}$ are:

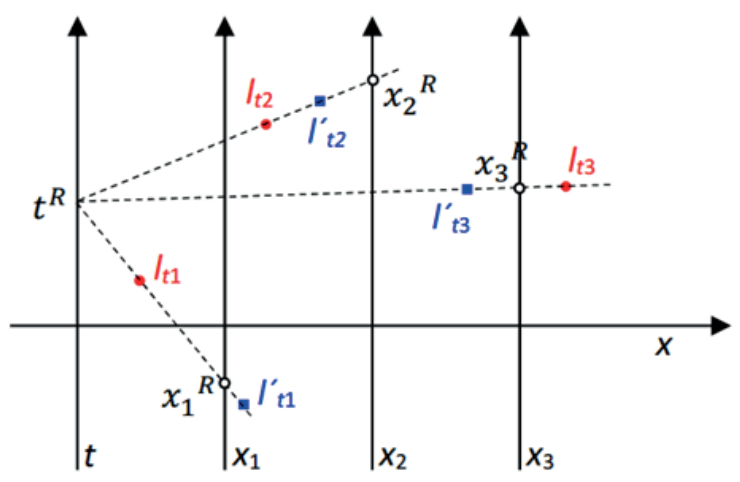

Figure 2 Representation of intersecting lines $\left(l \cap l^{\prime}=R\right)$ in parallel coordinates $t, x_{1}, x_{2}, x_{3}$

$l_{t, i}\left(\frac{i}{1-m_{i}}, \frac{q_{i}}{1-m_{i}}, 1\right) \sim\left(i, q_{i}, 1-m\right), m_{i} \neq 1, i=1,2,3$.

Let there be two lines $l, l^{\prime}$ with Equations (3):

$$
\begin{array}{rr}
l_{t, 1}: x_{1}=m_{1} t+q_{1} & l_{t, 1}^{\prime}: x_{1}=m_{1}^{\prime} t+q_{1}^{\prime} \\
l: & l_{t, 2}: x_{2}=m_{1} t+q_{2} \quad l^{\prime}: l_{t, 2}^{\prime}: x_{2}=m_{1}^{\prime} t+q_{2}^{\prime} . \\
& l_{t, 3}: x_{3}=m_{3} t+q_{3} \quad l_{t, 3}^{\prime}: x_{3}=m_{3}^{\prime} t+q_{3}^{\prime}
\end{array}
$$

For many interpretations, let $t$ denote the elapsed time and $\left(x_{1}, x_{2}, x_{3}\right)$ the space coordinates of a particle moving with constant velocity $\vec{v}$ and initial position $P$. The line $l$ is the trajectory of the particle 1 with velocity $\vec{v}=\left(m_{1}, m_{2}, m_{3}\right)$ and initial position $\left(q_{1}, q_{2}, q_{3}\right)$ and the line $l^{\prime}$ is the trajectory of the particle 2 with velocity $\vec{v}=\left(m_{1}^{\prime}, m_{2}^{\prime}, m_{3}^{\prime}\right)$ and initial position $\left(q_{1}^{\prime}, q_{2}^{\prime}, q_{3}^{\prime}\right)$. Lines $l, l^{\prime}$ are represented in parallel coordinates by points $l_{t, 1}, l_{t, 2}, l_{t, 3}$ and $l_{t, 1}^{\prime}, l_{t, 2}^{\prime}, l_{t, 3}^{\prime}$.

Lines $l, l^{\prime}$ have an intersection point $R\left(l \cap l^{\prime}=R\right)$, if all lines $l_{t, i} l_{t, i}^{\prime}, i=1,2,3$, intersect $t$ axis in the same point. This point corresponds to the $t$-coordinate of the intersection point $R$ (Figure 2). Numerically, let the lines have Equations (5), so one can easily see that the intersection point exists, if

$-\frac{q_{1}^{\prime}-q_{1}}{m_{1}^{\prime}-m_{1}}=-\frac{q_{2}^{\prime}-q_{2}}{m_{2}^{\prime}-m_{2}}=-\frac{q_{3}^{\prime}-q_{3}}{m_{3}^{\prime}-m_{3}}=t^{R}$.

Lines $l, l^{\prime}$ are parallel, if vectors $\vec{m}=\left(m_{1}, m_{2}, m_{3}\right)$, $\overrightarrow{m^{\prime}}=\left(m_{1}^{\prime}, m_{2}^{\prime}, m_{3}^{\prime}\right)$ are linearly dependent. The representation of parallel lines $l, l^{\prime}$ are points $l_{t, i}, l_{t, i}^{\prime}, i=1,2,3$ which have the same Cartesian coordinate $x$. Representation in parallel coordinates easily shows gaps on the $x_{1}, x_{2}, x_{3}$ axes at the same time $t^{c}$ in Figure 3.

Let there be two lines $l, l^{\prime}$, which are represented in parallel coordinates by points $l_{t, 1}, l_{t, 2}, l_{t, 3}$ and $l_{t, 1}^{\prime}, l_{t, 2}^{\prime}, l_{t, 3}^{\prime}$. Numerically, let the lines have Equations (5), then denote

$t_{1}=-\frac{q_{1}^{\prime}-q_{1}}{m_{1}^{\prime}-m_{1}}, t_{2}=-\frac{q_{2}^{\prime}-q_{2}}{m_{2}^{\prime}-m_{2}}, t_{3}=-\frac{q_{3}^{\prime}-q_{3}}{m_{3}^{\prime}-m_{3}}$.

If any $t_{i} \neq t_{j} ; i, j \in\{1,2,3\}$ then lines are nonintersecting (Figure 4). The absolute value $\left|\max t_{i}-\min t_{i}\right|$ converges to zero as the minimum distance $D$ between the lines converges to zero (when the two lines intersect, $D=0$ ). 


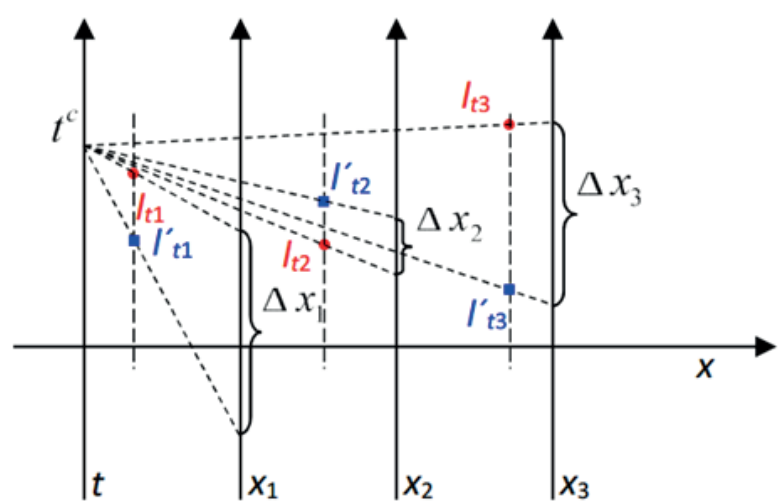

Figure 3 Representation of the parallel lines $l, l^{\prime}$ in parallel coordinates $t, x_{1}, x_{2}, x_{3}$

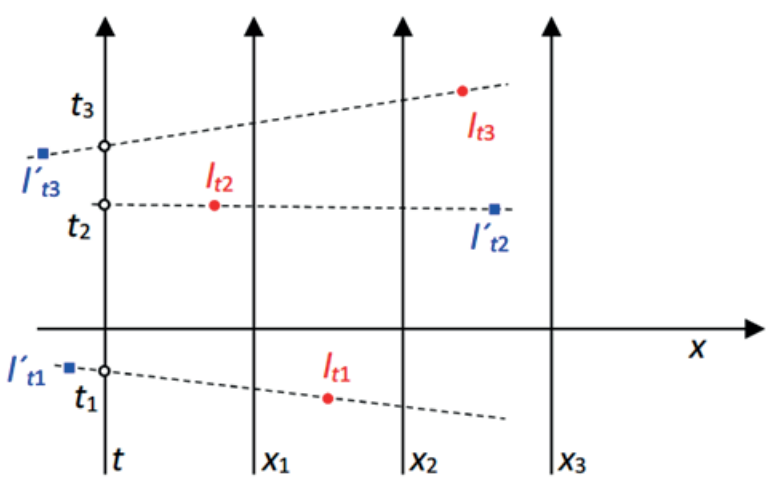

Figure 4 Representation of nonintersecting lines in parallel coordinates $t, x_{1}, x_{2}, x_{3}$

\subsection{Minimum distance between the two lines in space-time}

It is an interesting question to visualize the minimum distance between the two lines and points on each line where the minimum occurs. In many problems what is required is to minimize the distance when one or more of the variables are constrained to the same value for both lines. An example is air traffic control and motion planning in general, where knowing time and position, when two aircrafts are the closest, is needed. Let there be a point $P\left(t^{P}, x_{1}^{P}, x_{2}^{P}, x_{3}^{P}\right), P \in l$ and the point $P^{\prime}\left(t^{P^{\prime}}, x_{1}^{P^{\prime}}, x_{2}^{P^{\prime}}, x_{3}^{P^{\prime}}\right), P^{\prime} \in l^{\prime}$. Sometimes it is used Manhattan distance between points which is the sum of the lengths of the projections of the line segments between the points onto the coordinate axes [12]. The time is often constrained $\left(t^{P}=t^{P^{\prime}}\right)$ and one wants to find the minimum Manhattan distance between the points $P, P^{\prime}$ at that time:

$$
L_{1}\left(P, P^{\prime}\right)=\sum_{i=1}^{3}\left|x_{i}^{P}-x_{i}^{P^{\prime}}\right|
$$

If lines $l, l^{\prime}$ are parallel, one can see the visualization of gaps on axis at time $t^{c}$ in Figure 3. If lines $l, l^{\prime}$ are nonparallel and nonintersecting one can see in Figure 5 the gap on the $x_{1}$ axis (altitude) at time $t^{c}$. One may easily recognize (Figure 6) the gap on the $t$ axis when the coordinate $x_{1}$ is fixed to $x_{1}^{c}$ (altitude).

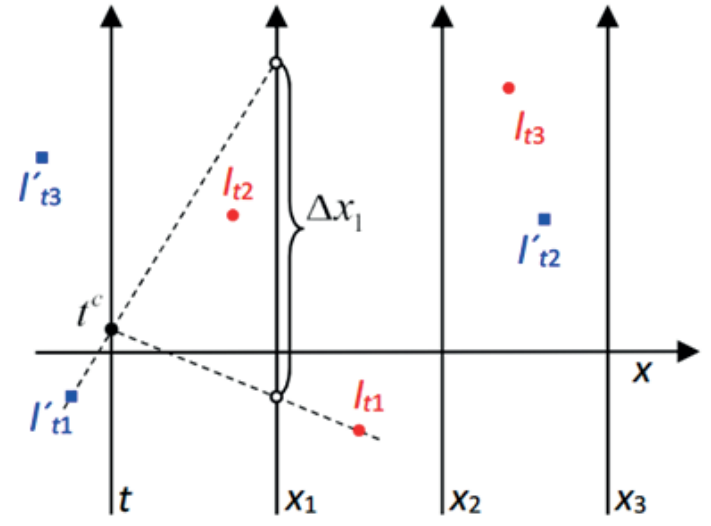

Figure 5 Representation of nonintersecting lines in parallel coordinates $t, x_{1}, x_{2}, x_{3}$

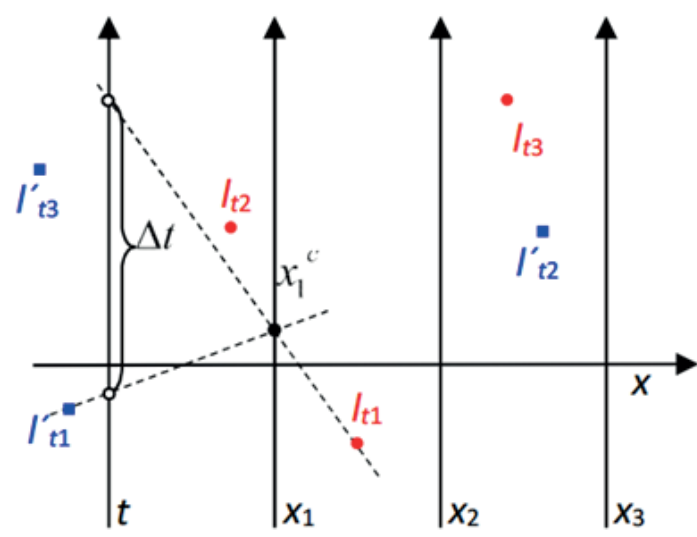

Figure 6 Representation of nonintersecting lines in parallel coordinates $t, x_{1}, x_{2}, x_{3}$

If the time is not fixed, the Manhattan distance between points $P, P^{\prime}$ is:

$L_{1}\left(P, P^{\prime}\right)=\left|t^{P}-t^{P^{\prime}}\right|+\left|x_{1}^{P}-x_{1}^{P^{\prime}}\right|+\left|x_{2}^{P}-x_{2}^{P^{\prime}}\right|+\left|x_{3}^{P}-x_{3}^{P^{\prime}}\right|$.

Using expressions (7) for points on lines one can write:

$$
\begin{aligned}
L_{1}(t) & =\sum_{i=1}^{3}\left|x_{i}^{P}(t)-x_{i}^{P^{\prime}}(t)\right|= \\
& =\sum_{i=1}^{3}\left|m_{i}-m_{i}^{\prime}\right|\left|t+\frac{q_{i}-q_{i}^{\prime}}{m_{i}-m_{i}^{\prime}}\right|
\end{aligned}
$$

denote $m_{i}-m_{i}^{\prime}=\Delta m_{i}$ and $t_{i}$ are intercepts defined in Equation (6)

$L_{1}(t)=\sum_{i=1}^{3}\left|\Delta m_{i} \| t-t_{i}\right|$

The unique minimum value of $L_{1}(t)$ is attained at $t=t_{i}$ for at least one $i=1,2,3$. The proof of this theorem one can find in [9] p. 93-96.

\subsection{Illustrative example}

Let there be two moving particles. The line $l$ is the trajectory of the particle 1 with velocity $\vec{v}=\left(\frac{1}{2}, \frac{1}{2}, \frac{1}{2}\right)$ and initial position 


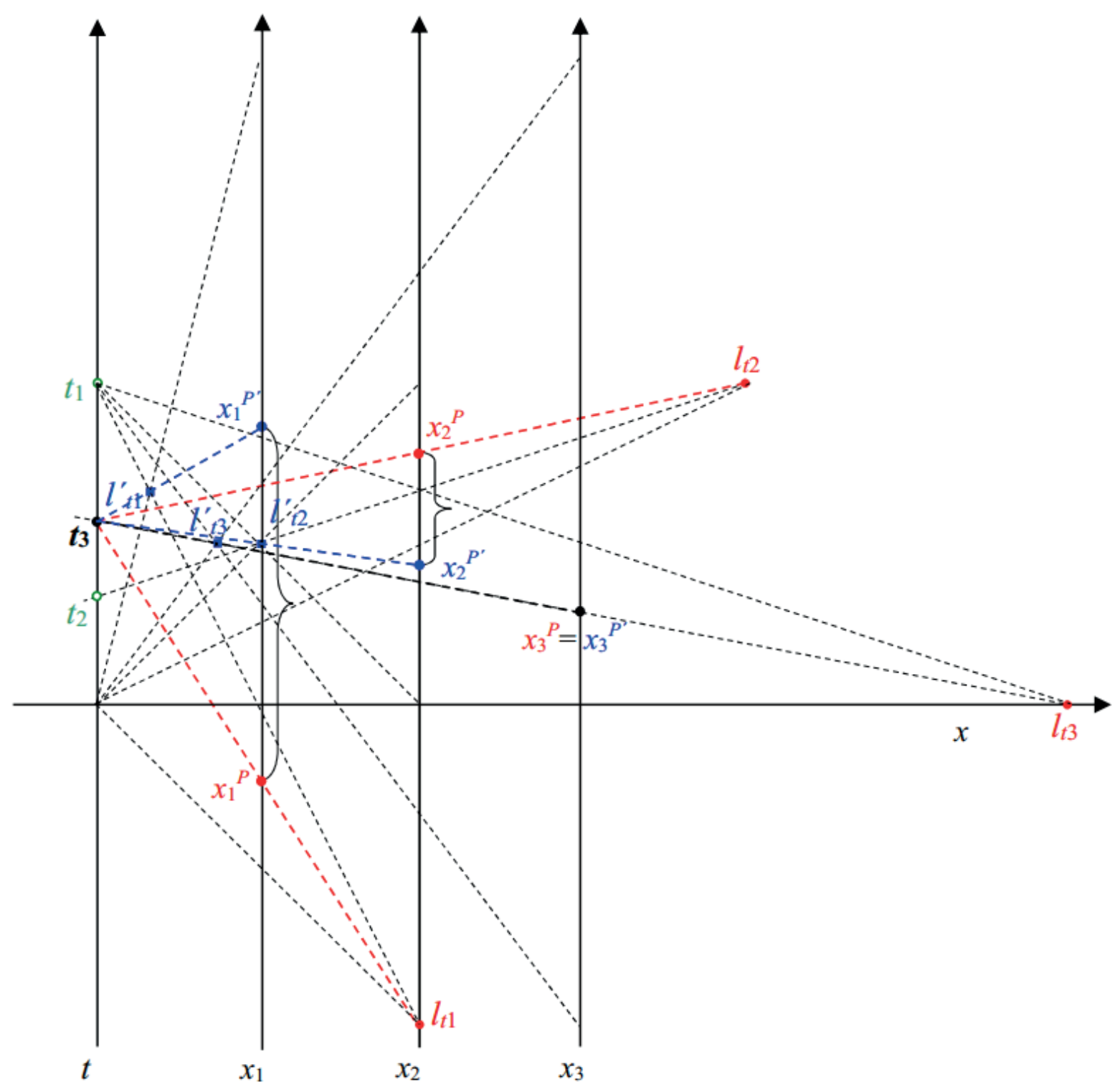

Figure 7 Minimal distance between lines

$(-1,1,0)$ and the line $l^{\prime}$ is the trajectory of the particle 2 with velocity $\vec{v}=(-2,-1,-3)$ and initial position $(4,2,4)$, see Equation (3), Figure 7:

$$
\begin{aligned}
& l: x_{1}=-1+t / 2, x_{2}=1+t / 2, x_{3}=t / 2, \\
& l^{\prime}: x_{1}=4-2 t, x_{2}=2-t, x_{3}=4-3 t .
\end{aligned}
$$

The lines are nonparallel and according to Equation (6) one obtains $t_{1}=2, t_{2}=\frac{2}{3}, t_{3}=\frac{8}{7}$.

The Manhattan distances for $t_{1}$ are:

$$
\begin{aligned}
& L_{1}\left(t_{1}\right)=0+2+3=5, \\
& L_{1}\left(t_{2}\right)=10 / 3+0+5 /+=5, \\
& L_{1}\left(t_{3}\right)=15 / 7+5 / 7+0=20 / 7 .
\end{aligned}
$$

The minimum Manhattan distance 20/7 occurs at time $t_{3}=8 / 7$.
The line $l$ is given by points $(0,-1,1,0),(2,0,2,1)$. The line $l^{\prime}$ is given by points $(0,4,2,4),(2,0,0,-2)$. Lines are represented in the parallel coordinates by points $l_{t, 1}, l_{t, 2}, l_{t, 3}$ and $l_{t, 1}^{\prime}, l_{t, 2}^{\prime}, l_{t, 3}^{\prime}$. Values of $t_{1}, t_{2}, t_{3}: t \cap l_{t, 1} l_{t, 1}^{\prime}=t_{1}$, $t \cap l_{t, 2} l_{t, 2}^{\prime}=t_{2}, t \cap l_{t, 3} l_{t, 3}^{\prime}=t_{3}$. Coordinates of points $P, P^{\prime}$ (points of the minimal Manhattan distance) are obtained: $x_{i}^{P^{\prime}}=x_{i} \cap t_{i} l_{t, i}^{\prime}, \quad i=1,2,3$, and $x_{i}^{p^{\prime}}=x_{i} \cap t_{i} l_{t, i}^{\prime}, i=1,2,3$. The minimum Manhattan distance is the sum of lengths of particular line segments on axis $x_{1}, x_{2}, x_{3}$.

\section{Discussion and conclusion}

The aim of this paper was to give the basic information on parallel coordinates and possibility of their using in air traffic control. Equations of lines are expressed in the time-space and stated conditions for their position illustrated by an example of finding the minimum distance between two nonintersecting lines. 
Parallel coordinates may represent multidimensional data in wide range of applications: data mining (USA patent), computer vision (USA patent), as well as optimization, process control, clustering and classification problems and more recently in intrusion detection and elsewhere [3]. ParallAX is introduced as a new user-friendly tool for visualization and analysis of multivariate data using the parallel coordinates methodology [13]. Other possibilities of visualization via parallel coordinates are discussed in [14-16]. The increasing popularity of the parallel coordinates may be illustrated by the number of publications with the term "parallel coordinates" present in their title: It has been rising steadily from 14 in the year 1991 to approximately 543 in 2011, with a total of 5620 publications as reported by Google scholar on the 15th of December, 2012 [17]. While there is a large amount of papers about parallel coordinates, there are only a few notable software publicly available to convert databases into parallel coordinates graphics, e.g. XDAT, XLSDAT.

\section{Acknowledgements}

Publication of this paper was supported by the Centre of excellence for systems and services of intelligent transport II., ITMS 26220120050 supported by the Research \& Development Operational Programme funded by the ERDF.

\section{References}

[1] FREUDENRICH, C. How air traffic control works. In: How stuff works science [online]. [accessed 2017-05-05]. Avalaible from: http://science.howstuffworks.com/transport/flight/modern/ air-traffic-control.htm

[2] D’OCAGNE, M. Parallel and axial coordinates [online]. Paris: Gauthier-Villars, 1885 [accessed 2017-05-05]. Avalaible from: http://archive.org/stream/coordonnespara 100ocaggoog \#page/n9/mode/2up

[3] INSELBERG, A. Parallel Coordinates: visual multidimensional geometry and its applications. Dordrecht: Springer, 2009. ISBN 978-0-387-21507-5.

[4] WEGMAN, E. J. Parallel-coordinate plots and density plots. Encyclopedia of statistical sciences. New Jersey: John Wiley \& Sons, 2004. ISBN 978-0-471-15044-2.

[5] GNAP, J., KAlASOVA, A., GOGOLA, M., ONDRUS, J. The Centre of Excellence for transport service and control. Communications - Scientific Letters of the University of Zilina [online]. 2010, 12(3A), p. 116-120. ISSN 1335-4205, eISSN 2585-7878. Available from: http://komunikacie.uniza.sk/index.php/communications/article/view/956

[6] GLENDENNING, K., WISCHGOLL, T., HARRIS, J., VICKERY, R., BLAHA, L. Parameter space visualization for large-scale datasets using parallel coordinate plots. Electronic Imaging [online]. 2016, 60(1), p. 10406-1-10406-8(8). ISSN 2470-1173. Available from: https://doi.org/10.2352/J.ImagingSci.Technol.2016.60.1.010406

[7] PELTONEN, J., LIN, Z. Parallel coordinate plots for neighbor retrieval. In 12th International Joint Conference on Computer Vision, Imaging and Computer Graphics Theory and Applications : proceedings [online]. Vol. 3. IVAPP. 2017. ISBN 978-989-758228-8, p. 40-51. Available from: https://doi.org/10.5220/0006097400400051

[8] LI, J., MARTENS, J. B., WIJK, J. J. Judging correlation from scatterplots and parallel coordinate plots. Information Visualization [online]. 2010, 9(1), p. 13-30. ISSN 1473-8716, eISSN 1473-8724. Available from: https://doi.org/ 10.1057/ivs.2008.13

[9] COHAN, S. M. Mobility analysis of mechanisms through the parallel coordinate system. Mechanism and Machine Theory [online]. 1986, 21(1), p. 63-71. ISSN 0094-114X, eISSN 1873-3999. Available from: https://doi.org/10.1016/0094-114X(86)90030-3

[10] OPACH, T., ROD, J. K. Do choropleth maps linked with parallel coordinates facilitate an understanding of multivariate spatial characteristics? Cartography and Geographic Information Science [online]. 2014, 41(5), p. 413-429. ISSN 1523-0406, eISSN 1545-0465. Available from: https://doi.org/10.1080/15230406.2014.953585

[11] STEED, CH. A., FITZPATRICK, P. J., SWAN, J. E., JANKUN-KELLY, T. J. Tropical cyclone trend analysis using enhanced parallel coordinates and statistical analytics. In: Cartography and Geographic Information Science [online]. 2009 [accessed 2019-0304]. Available from: https://pdfs.semanticscholar.org/259f/276799e697441424fd3d6be476e4a46e6381.pdf

[12] MUNOZ, D., LARA, F. B., VARGAS, C., ENRIQUEZ-CALDERA, R. Position Location Techniques and Applications. Academic Press, 2009. ISBN 978-0-123-74353-4.

[13] AVIDAN, T., AVIDAN, S. ParallAX - A data mining tool based on parallel coordinates In: Computational Statistics [online]. Physica-Verlag, 1999, 14(1), p.79-89. ISSN 0943-4062. Available from: https://doi.org/10.1007/PL00022707

[14] VAIDYA, M. Survey of parallel data processing in context with MapReduce. Computer Science and Information Technology [online]. 2011, 1, p. 69-80. ISSN 2231-5403 Available from: https://doi.org/10.5121/csit.2011.1307

[15] ONDRUS, J., DICOVA, J. Potential of prediction quantification and trends in transport requirements as tool of transport management and development. Transport and Telecommunication [online]. 2013, 14(4), p. 316-324. ISSN 1407-6160. Available from: https://doi.org/10.2478/ttj-2013-0027

[16] OPACH, T., ROD, J. K. Augmenting the usability of parallel coordinate plot: The polyline glyphs. Information Visualization [online]. 2018, 17(2), p. 108-127. ISSN 1473-8716, eISSN 1473-8724. Available from: https://doi.org/10.1177/1473871617693041

[17] HEINRICH, J., WEISKOPF, D. State of the art of parallel coordinates. In: Eurographics [online]. 2013 [accessed 2019-03-04]. Avalaible from: http://joules.de/files/heinrich_state_2013.pdf 